KaRL VON FrISCH's Nobel Prize winning discovery of the dance language of honeybees is one of the classics of biology. Over many years von Frisch collected evidence to show that when a successful foraging bee returns to the hive it is able to recruit other workers by communicating the precise distance and direction to the food source, encoding this information in the abstract symbolism of a 'dance' performed on the hive surface. The distance is encoded in the speed of dancing and the direction by the orientation of the figure-ofejght dance in relation to gravity. This remarkable feat places honeybees second only to man and perhaps chimpanzees in the use of abstract symbols in transmission of information.

About eight years ago, Adrian Wenner pointed out that two of von Frisch's best known experiments were not as convincing a demonstration of the dance language as most people had thought. In these two studies, known as the 'step' and 'fan' experiments, von Frisch showed that naive recruit bees would go specifically to experimental food dishes indicated by the dances of experienced foragers, and ignore identical nearby control dishes. In the fan experiment the control and experimental dishes were at the same distance from the hive but in different directions, and in the step experiment the dishes were in the same direction but at different distances. Wenner correctly pointed out that not only is the experimental dish in these experiments unique in being the one indicated by the dance, but also it is the one towards which experienced foragers are flying. The recruits could either be using the dance information or simply watching where the foragers go. Wenner eliminated this confounding variable by training equal numbers of foragers from a separate (control) hive to go to each of the control dishes, and he now found that recruits from the experimental hive no longer preferred the dish indicated by the dance. Wenner's conclusion from this and other experiments was that recruits may be stimulated to leave the hive by the dance, but that they normally search for food by smell, using the smell of the dancing foragers as a guide.

Naturally enough, Wenner's results aroused enthusiastic criticism, followed by new experiments supporting

\section{The bee language controversy}

\author{
from John R. Krebs
}

von Frisch's story, with further counterclaims by Wenner and his colleagues, culminating in a headline in Nature $\mathbf{J} 241,171$; 1973) suggesting that von Frisch's data could be explained without recourse to the dance language theory. One valuable consequence of Wenner's criticisms was that people devised more rigorous tests of bee dance communication, and the latest of these studies, by J. L. Gould (Science, 189, 685; 1975) finally shows beyond doubt that bees use the dance information.

In a most beautifully ingenious experiment, Gould exposed recruit bees to conflicting information: the dance indicated one direction, while the foragers that gave the dance flew in another direction. The key to Gould's experiment is the fact that bees can be persuaded to orientate their dance in relation to the Sun or (in the case of Gould's experiment) a bright light instead of gravity. As long as the recruits can also see the Sun or bright light, they automatically 'read' the dance correctly, using the light source instead of gravity as a reference. What Gould did then was to cover the ocelli of the dancing foragers with black paint so that they could not see the artificial Sun (a 650 watt bulb) shining into the hive but could still forage and dance. The returning foragers now oriented their dance with respect to gravity, as usual in a dark hive, but the recruits interpreted the dance as though it were oriented with respect to the bright light. To give an example, suppose the bright light is placed at $60^{\circ}$ to the vertical, and a returning forager has just come from a food dish directly in line with the real Sun outside. The forager dances on the vertical surface of the hive, unaware of the bright light, and orientates her dance vertically upwards (which is the gravity-based code for 'towards the Sun'). The recruits, however, read the information as ' $60^{\circ}$ from the line of the Sun', and head off in a different direction from the foragers. Recruits always arrived at food dishes in the direction indicated by their interpretation of the dance, and not at the experimental dish used by the foragers. Further, by moving the bright light to different positions in the hive, Gould could get the recruits to go to different feeding dishesalways the one predicted by incorrect reading of the dance.

It is also particularly satisfying that Gould was able to show at least one reason why Wenner did not find any effect of the dance on recruits. In the initial part of any dance experiment, a few chosen foragers are trained to fly to a particular dish, this being the dish they indicate to naive recruits in the dance. Wenner used training dishes scented with the same odour as was later used in the experiment, while Gould was careful to change to a new scent on the day of the experiment. If Gould used the same scent for both training and experiment, he got the same results as Wenner: the recruits went to any dish with the same scent as that carried into the hive by foragers. The conflict between the von Frisch and Wenner results is at least partly due to details of experimental design and, as Gould points out, this might well mean that recruit bees use the dance information when they are just starting to exploit a new food source but use scent, as Wenner proposed, when exploiting the same food source at a later stage. indoleamines to $\mathrm{N}$-methylated products with hallucinogenic properties. Traces of both the mono- and dimethylated derivatives of tryptamine and serotonin are excreted in human urine and may occasionally be found in blood; the excretion, moreover, does not seem to be derived from the diet or bacterial action in the bowel. There are also reports, as yet unconfirmed and inadequately documented, that mentally ill patients with the diagnosis of non-affective psychosis tend to excrete more DMT in their urine than do mentally ill patients with other diagnoses or normal subjects. It is, however, uncertain how much, if any, of urinary DMT is derived from tryptamine methylated in the brain. There is no doubt that human lung contains an SAM-dependent methyltransferase which forms DMT from tryptamine, the enzymic basis for the same reaction in mammalian brain is now much less clear than formerly suspected. Earlier reports on the occurrence of an indoleamine methyltransferase in brain using SAM as methyl donor were based on unsatisfactory methods of product identification and the reaction urgently needs reassessing by more precise methods such as gas chromatography and mass spectrometry.

Suspicions that folic acid might be involved in the endogenous formation of hallucinogens in the body arose about 3 years ago when several reports appeared indicating that besides SAM, 